\title{
Review of Methods for the Excitation of Atomic and Ionic Spectra by Means of High-Frequency Discharges and Sliding Sparks
}

\author{
Lennart Minnhagen ${ }^{1}$
}

(May 26, 1964)

\begin{abstract}
Two groups of excitation techniques useful for the production of ionic spectra are reviewed, namely high-frequency excitation and the sliding spark. The development of the arrangements is described and their characteristics given as regards power generation, electrodes, and spectroscopic features. The ionization stages obtained by each method of excitation are considered.
\end{abstract}

\section{Introduction}

The development of appropriate sources for the excitation of atomic and ionic spectra is a fundamental task in atomic spectroscopy. The demands on the sources are, generally speaking, that they should produce the actual spectra with a high degree of completeness, with the best possible line quality, and, as far as possible, free from impurities. Further, the sources should make it possible to decide from which ionization stage the spectrum lines originate. The required sharpness of the lines depends on the character of the investigation. Obviously the greatest possible reduction of the line width-caused by Doppler effect, collisions, Stark effect etc. - is necessary for the determination of highly accurate wavelengths and for the investigation of hyperfine structure by means of highresolution spectroscopy, while the demands for sharpness are much less in some kinds of straightforward analytical work. It is sometimes an advantage to have excitation conditions which give observable Stark-effect broadenings, since the broadenings depend on the quantum properties and energies of the levels involved and thus facilitate the analysis. Analogously, light sources giving rise to strong self-reversal are inappropriate in some investigations, whereas they may be advantageous in the analysis of a spectrum.

In the present review two groups of sources useful for the production of spectra of low and medium ionization stages will be treated, namely highfrequency excitation and the sliding spark.

\section{High-Frequency Excitation}

The method of exciting spectra by means of a highfrequency discharge is quite old. Up to about 1930 , spark-generated high-frequency oscillations, which are strongly damped, were used almost exclusively for exciting spectra; more recently the enormous

On leave of absence from Lund Institute of Technology, Lund 7, Sweden. progress in the field of transmitter technique has made possible the excitation of spectra by means of continuous oscillations, even in the form of pulsed oscillations, over a very wide frequency range.

As regards the transfer of high-frequency power to the contents of the discharge tube, four experimental arrangements are utilized:

1. Internal electrodes

2. External electrodes

3. Coil surrounding the discharge tube

4. Discharge tube in a cavity resonator.

Some examples of typical experimental arrangements and their excitation properties are given below.

\subsection{Spark-Generated Oscillations}

\section{a. Internal Electrodes}

The original method for generating high-frequency oscillations is to use a circuit consisting of a condenser, a coil, and a spark gap, and feeding it by a-c or d-c high voltage. Along these lines de Bruin $[1,2]^{2}$ and Humphreys [3], among others, have worked with much success on the spark spectra of the inert gases. In a typical coupling de Bruin used a $0.02-\mu \mathrm{F}$ oil condenser in the $\mathrm{HF}$ circuit fed from a $25-\mathrm{kV}$ transformer which in turn was connected to the a-c mains via a variable transformer. The discharge tube was of the Geissler type with a quartz capillary $10 \mathrm{~cm}$ long and with a $3 / 4 \mathrm{~mm}$ inner diameter. The hollow aluminum electrodes were connected to the coil. In one of his papers on the spark spectra of neon, de Bruin [1] reports that the highest intensity was obtained in the capillary at a rather high pressure, namely $2 \mathrm{~cm} \mathrm{Hg}$. The following ionization stages have been attained: Ne vi [4], Ar iv [5], Kr Iv [6], Xe Iv [7]. The association of the various lines of a spectrum with the different ionization stages may be done by variation of the charging voltage and the inductance. There seem, however, to be some difficulties in distinguishing the higher ionization stages.

2 Figures in brackets indicate the literature references at the end of this paper. 
Drawbacks of this excitation method are that the electrodes can give impurity lines; further, the inner walls of the narrow capillary are attacked, particularly at high excitations and, under certain circumstances, a quartz capillary can give an impurity spectrum which is dominating and which masks the lines of the element under investigation, over extended wavelength ranges.

\section{b. Electrodeless Discharge}

One of the first to investigate the spark-generated electrodeless high-frequency discharge was J. J. Thomson [8]. This type of discharge was later used for the excitation of spectra. The high-frequency oscillations may be generated as described in the previous section. A large number of spectroscopic investigations have been carried out by this method, the pioneer work being to a great extent due to E. and L. Bloch and their co-workers. In a typical experiment [9] they used a quartz or Pyrex discharge tube having a length of $20 \mathrm{~cm}$ and a diameter of $30 \mathrm{~mm}$-in other cases they have used shorter tubes. The tube is surrounded by a coil containing 10 to 12 turns of 1.5-mm copper wire. The coil, a condenser, and a spark gap form a high-frequency circuit which is fed from a high-voltage transformer working at $42 \mathrm{c} / \mathrm{s}$. A high-frequency of about $400 \mathrm{kc} / \mathrm{s}$ was used in investigating the spark spectra of mercury; in obtaining the spark spectra of $\mathrm{Ar}, \mathrm{Kr}$, and $\mathrm{Xe}$, gas pressures of $0.6,0.3$, and 0.9 torr $\left(\mathrm{mm} \mathrm{Hg}^{\mathrm{s}}\right)$, respectively, were used [10]. The so-called electrodeless ring discharge obtained in this way has the important property of permitting the separation of spectra from different ionization stages with some certainty, since the ionization stage excited is a function of the coordinates of the ions. If the common axis of the discharge tube and the coil coincides with the axis of a spectrograph (end on observation), then the spectrum appears as follows when a stigmatic spectrograph is used: At a certain breakdown voltage, depending on the dimension of the spark gap, certain lines have an intensity which does not vary much along the line or which is strongest at the center; they emanate from neutral atoms. Other lines indicate low intensity at the axis of the discharge tube and increasing intensity towards the walls of the tube; they are emitted by ionized atoms. The separation of different ionization stages is based mostly on a study of the intensity distribution along the lines. An increased spark gap, which means an increased breakdown voltage, results in a decreased intensity at the ends of the arc lines, the intensities of which are more and more concentrated at the center of the lines, whereas the spark lines get stronger towards the ends of the lines. At sufficiently strong excitations, new "short" lines appear, apparently emitted by higher ionized atoms near the walls of the discharge tube. The authors report that they have been able to a great extent to separate Hg II-IV, Ne II-III, Ar II-IV, Kr II-IV, Xe II-IV, etc. Impurity lines often show characteristic features by means of which they can be recognized.

Boyce's [11-14] well-known investigations of the spark spectra of the inert gases in the vacuum ultraviolet were performed by means of electrodeless highfrequency excitation; he has attained the following ionization stages: Ne IV, $\mathrm{Ar} \mathrm{v}, \mathrm{Kr}$ IV, Xe IV. It should be noticed, however, that the lines observed for the highest ionization stages are those most easily excited, namely the combinations of the lowest excited levels with the ground term. Since the measurements are confined to the vacuum ultraviolet the observations tell little about the excitation of levels of high angular momentum.

A similar excitation method was used by Eriksson [15] in investigating $\mathrm{N}$ II. The quartz discharge tube, $18 \mathrm{~cm}$ long and with a central cylindrical part having $15 \mathrm{~mm}$ inner diameter, was surrounded by a coil made of silvered copper wire. With a $0.007 \mu \mathrm{F}$ capacitor the frequency was about $700 \mathrm{kc} / \mathrm{s}$. In some experiments a $0.014 \mu \mathrm{F}$ capacitor was used. The optimum conditions for developing the $\mathrm{N}$ II spectrum were obtained at a nitrogen pressure of 0.05 to 0.07 torr - which was found to be rather critical-and a spark voltage of 24 to $36 \mathrm{kV}$. The admission of helium to the discharge tube was found to bring about an increased excitation along the axis of the tube, particularly in its central part. The discharge in the nitrogen-helium mixture gives $\mathrm{N}$ II lines which have nearly the same intensity along the lines, whereas the $\mathrm{N}_{\mathrm{I}}$ lines are weak at the center and the N III lines have strong central parts. Thus the appearance of lines belonging to different ionization stages is reversed in relation to the one observed by L. and E. Bloch, but it is in accordance with that observed by Esclangon. From a spectroscopic point of view the important feature certainly is that lines from different ionization stages have different characteristics.

The absence of electrodes contributes to the purity of the spectrum. Further, since high intensities can be obtained even in a wide discharge tube, the walls are much less attacked than those of Geissler tubes with inner electrodes.

A particular form of excitation is the theta-pinch discharge. A light source, recently described by Beckasten, Hallin, Johansson, and Tsui [16], consists of a Pyrex tube $50 \mathrm{~cm}$ long, $6 \mathrm{~cm}$ in diameter, surrounded by a single turn loop through which a 6.6 $\mu \mathrm{F}$ condenser bank is discharged. The voltage can be chosen between 7 and $25 \mathrm{kV}$, and gas pressures between 0.006 and 0.025 torr have been used. A characteristic of the design is a low inductance, which means that fairly high ionization stages can be obtained; investigations of the $\mathrm{O}$ III-VI and the $\mathrm{N}$ III-VI spectra are in progress. ${ }^{3}$

\subsection{Continuous Radiofrequency Oscillations and High-Frequency Pulses}

By means of modern transmitter technique it is possible to generate high-frequency oscillations over a very large frequency and power range. Continuous oscillations as well as high-frequency pulses are useful for the excitation of spectra.

3 Spectroscopic features of the large installations for the production of magnetically compressed plasma are not within the scope of the present review. 
Among early investigations with continuous wave oscillators, the work of Esclangon [17] should be mentioned. With a $6-\mathrm{kV}$ anode voltage on the final tube, $3 \mathrm{~kW}$ could be generated at frequencies between 2 and $6 \mathrm{Mc} / \mathrm{s}$. The commercial therapeutical apparatus used by Gatterer and Frodl [18] has 600 $\mathrm{W}$ power at frequencies between 40 and $100 \mathrm{Mc} / \mathrm{s}$. At frequencies of the above magnitude, the highfrequency power can be transferred to the contents of the discharge tube either by outer electrodes in contact with the outer walls of the discharge tube or by a coil surrounding it. In the former case a displacement current appears in the tube. The current density is, however, low at radio frequencies, if the cross section of the tube is large. For ordinary spectroscopic purposes the transfer by means of a coil is suitable. Although the discharge process is complicated, it may be presumed that the dominating excitation is due to impact with electrons, which are accelerated in the high-frequency magnetic field generated in the coil. The electrons should, therefore, have a strong tendency to move in curved paths which are perpendicular to the axis of the surrounding coil. A careful investigation of the discharge process is needed from a spectroscopic point of view, since a better theoretical basis is required for the development of the high-frequency excitation sources.

The discharge can be maintained at low gas pressures in the tube, which contributes to the emission of sharp lines; in fact, the line quality can be good enough to allow interferometer measurements. The low pressure is also an advantage for work in the vacuum ultraviolet, in the wavelength ranges where no transparent windows exist; the leakage of gas through the slit is necessarily lower when the pressure in the discharge tube is low. Low pressures in the source and spectrograph are particularly required in investigating resonance lines of neutral atoms in order to limit self-reversal. The absence of electrodes contributes to a spectrum free from impurity lines. Continuous high-frequency oscillations give only low ionization stages; usually, the first spectrum, and-more or less complete the second spectrum are excited. The excitation degree depends, of course, on the power transferred to the discharge and also on the nature of the atoms excited. Molecular spectra are easily excited, which means that the discharge tube and the circulating system must be clean and contain good cooling traps in order to reduce the appearance of impurity bands. Experience seems to indicate that in itself the frequency of the oscillations has no marked influence on the excitation. It might, however, have a secondary influence since its magnitude determines the dimensions of circuits, coils, etc., which, in turn, partly determine the transfer of power to the discharge tube. Since very large high-frequency powers can be generated in a modern continuous wave transmitter, very brilliant light sources can be obtained. The transfer of high-frequency power is limited by the effectiveness of the method of cooling the discharge tube. This cooling is usually done by forced air because of the difficulties of arranging liquid cooling in the high-frequency field. Water cooling is inappropriate since the high-frequency losses are large.

The increase of the excitation of the second spectrum relative to the first spectrum, and the excitation of higher ionization stages, requires higher instantaneous powers at a mean power which is limited by the cooling conditions. This may be done by running an oscillator intermittently. By means of pulsing, it is possible to increase the plate voltage of the transmitting tubes well above the normal values. The anode voltage is limited by the high voltage insulation and the anode dissipation. As a rule the insulation permits a plate voltage much higher than that recommended for continuous operation. If the mean power does not exceed the power at continuous operation, then the average plate dissipation will not exceed allowed values, in spite of the high instantaneous power. Provided that the anode voltage is not raised so much that the anode current attains its saturation value, the instantaneous high-frequency power will be proportional to the square of the anode voltage. The pulsing procedure was introduced into experimental spectroscopy fairly late. Self-oscillating devices were used by Goudet, Herreng, and Nief [19], as well as by Roig and Buret [20]. In the first case the $150-\mathrm{Mc} / \mathrm{s}$ oscillator was pulsed by anode voltage peaks and in the latter case the $20 \mathrm{Mc} / \mathrm{s}$ oscillator was grid-modulated at $500 \mathrm{c} / \mathrm{s}$.

In a more elaborate arrangement described by Minnhagen and Stigmark [21], the oscillator is controlled by a pulse generator, which starts the oscillator at the beginning of a pulse and stops it at the end of the pulse. The oscillator frequency is multiplied and then fed into a driver which gives sufficient power for the final tube. Via a coupling link, the high-frequency power is transferred to a tuned circuit composed of a variable condenser and a coil surrounding the discharge tube. Various tube dimensions have been used; in some of the inert gas investigations the total length of the quartz tube was $19 \mathrm{~cm}$ with a central cylindrical part having $20 \mathrm{~mm}$ outer diameter. The pulse duration and the pulse frequency are adjustable within certain limits and the oscillator frequency between 7.8 and 9.6 $\mathrm{Mc} / \mathrm{s}$. With no pulse generator, the oscillator runs continuously. When the final tube runs continuously the anode voltage is $3 \mathrm{kV}$ and the anode dissipation $450 \mathrm{~W}$. For pulsed operation it is possible to increase the anode voltage to $9 \mathrm{kV}$ at a pulse frequency of about 500 pulses per sec and a pulse duration of $400 \mu \mathrm{sec}$. The average power is about $550 \mathrm{~W}$ under these circumstances and the estimated instantaneous power $3 \mathrm{~kW}$. Appropriate gas pressures for the excitation of the spark spectra of the inert gases were found to be 0.02 to 0.10 torr. The correct tuning of the electrical circuit associated with the discharge tube is important in order to get optimal loading, which means the transfer of large high-frequency powers to the contents of the discharge tube. In the case of argon the apparatus is able to excite Ar I, Ar II, and, partly, Ar III. By variation of the pulse power it is possible to separate, 
with a high degree of certainty, $\mathrm{Ar} \mathrm{II}$-which is developed in a very complete way-from $\mathrm{Ar}$ I and Ar III. There is no perceptible variation of the intensity and the degree of ionization along a diameter of the discharge tube. When the oscillator is run continuously, Ar I and parts of the Ar II spectrum appear. During pulsed operation, the Ar I intensity decreases when the pulse power is increased, whereas the $\mathrm{Ar}$ II spectrum develops and gets stronger. Further, Ar III gets more complete and gains intensity in relation to $\mathrm{Ar}$ II. If a line is weak even at the highest pulse powers attainable with the oscillator, it may be difficult to decide whether it belongs to $\mathrm{Ar}$ II or to $\mathrm{Ar}$ III. This decision would require still higher pulse powers, which have been generated by means of $25-\mathrm{kW}$ transmitting tubes $[22,23]$ run at $20-\mathrm{kV}$ anode voltage $(12 \mathrm{kV}$ is recommended when they are run continuously). Because of the high $d-c$ and rf voltages, special variable tubular condensers are required. One installation, containing a single final tube, has a high frequency which is adjustable around $9 \mathrm{Mc} / \mathrm{s}$, a pulse frequency adjustable between 40 and 900 pulses per sec, and a pulse duration that can be chosen between 80 and 1000 usec. It is a great problem to transfer the large pulse powers into the discharge tube; the best arrangement found is described in reference [22]. The highfrequency power is taken from the tank circuit of the power amplifier by means of a coupling coil and, via a coaxial conductor, fed to a separate tuning circuit containing a coil which surrounds the discharge tube. High quality insulations and large flashover distances are needed.

Another installation containing two final tubes in "push-push" coupling has an $18 \mathrm{Mc} / \mathrm{s}$ frequency but the doubled frequency has no marked influence on the excitation. Typical values of pulse duration and pulse frequency are $80 \mu \mathrm{sec}$ and 40 pulses per sec.

The high pulse power oscillators have been used to excite spark spectra of the inert gases. The argon investigation is, as yet, the most advanced one and part of it was performed by means of the single tube installation. When $\mathrm{Ar}$ II, $\mathrm{Ar}$ III, and Ar IV are excited, the variation of the line intensity with the pulse power permits a decisive separation of the ionization stages. The Ar IV lines appear only at the highest pulse powers. The situation is similar for xenon. The excitation of the spark spectra of neon, on the other hand, is much more difficult. This may be due to several causes. One probably is that the $\mathrm{Ne}$ I, II, and III ionization potentials are larger than those for the heavier inert gases.

Lines due to impurities can usually be recognized since they show a variation in intensity with the pulse power which differs from that of the element under investigation. Sometimes it is useful to employ pulsed operation even to produce spectra of neutral atoms, namely in spectral ranges which contain impurity bands, since an increased highfrequency power means a decreased intensity of the band lines.

A new construction of a self-excited oscillator (Stigmark [24]) has been tested for the excitation of spectra. The oscillator contains an industrial tube with such characteristics that the tube will withstand very large variation in loading conditions without being damaged. The tube has been used in a tuned plate, tuned grid circuit in which the anode tank consists of a cavity tuned to $27.12 \mathrm{Mc} / \mathrm{s}$. Because of the rather high $Q$ value of the cavity, even under heavy loading conditions, the frequency shift of this type of oscillator is very small and can easily be kept within narrow frequency limits $( \pm 0.6 \%)$. The power is transferred from the cavity to an outer, tuned discharge-tube circuit by means of link coupling. The power is about $15 \mathrm{~kW}$ at the normal anode voltage of $6 \mathrm{kV}$, the anode efficiency being 60 percent. By means of pulse techniques and a raised anode voltage, the output pulse power may be increased considerably.

\subsection{Electrodeless Microwave Excitation}

The electrodeless excitation of spectra by radio frequencies above $100 \mathrm{Mc} / \mathrm{s}$ and by microwaves, often in the 2000 to $3000 \mathrm{Mc} / \mathrm{s}$ range, has been very successful. Under proper conditions, very sharp and unperturbed lines are produced, useful even for high-precision interferometric work. However, for very accurate wavelength determinations the sources should be used with some caution since there are indications that they may give shifts of higher excited levels $[25,26]$.

Since continuous microwave oscillations do not produce higher ionization stages they will not be discussed in the present review but reference given to some useful articles (Meggers and Westfall [27], Jacobsen and Harrison [28], Zelikoff et al. [29], Corliss, Bozman, and Westfall [30], Tomkins and Fred [31], Worden, Gutmacher, and Conway [32]).

\section{Excitation by Means of Sliding Sparks}

The excitation of higher spark spectra has to a great extent been performed by means of vacuum sparks, which can give very high ionization stages. Because of the large voltages and current densities necessary, the lines may be broadened and shifted through Stark effect and this is particularly true for levels with large azimuthal quantum numbers. In this respect the sliding spark, suggested by Vodar and Astoin [33], is superior, since it can be operated at lower voltage than the vacuum spark. In the original arrangement of Vodar and Astoin a spark is produced which slides along the surface of a carbon rod provided with two ring electrodes connected to a condenser charged to a $30-\mathrm{kV}$ voltage. (For further developments by Vodar et al., see for example ref. [34].)

This idea was further developed by Bockasten $[35,36]$ for his investigation of $\mathrm{C}$ III and $\mathrm{C}$ IV. His arrangement, which is described in detail in reference [35], is essentially as follows. The source consists basically of two cylindrical graphite electrodes which are separated by a porcelain disk. The latter has a small bore along its axis through which the dis- 
charge takes place. This confinement of the discharge brings about a large gain in intensity. The discharge is viewed through a radial channel in the porcelain disk. In order to get proper working conditions it is important that the end surfaces of the electrodes fit very well to the surfaces of the disk. The light source works well at $10^{-3}$ torr and at lower pressures. 'To control the working' conditions it is necessary to have an external spark gap. The separation of different ionization stages is done by variation of the inductance in the spark circuit. The sliding spark works well even with a large inductance, which is not the case with the vacuum spark. Typical conditions for the excitation of $\mathrm{C}_{\text {III }}$ and $\mathrm{C}_{\mathrm{IV}}$ are: external spark gap 1.5 to $4.5 \mathrm{~mm}$ with corresponding spark voltages 6 to 15 $\mathrm{kV}$, spark frequency at $15 \mathrm{kV} 6$ to 8 sparks per second with a $0.3 \mu \mathrm{F}$ capacitance. The sliding spark spectra contain lines from the electrodes as well as from the insulating disk between them. In his first paper Bockasten reports tests with various metal electrodes: $\mathrm{Be}, \mathrm{Al}, \mathrm{Fe}, \mathrm{Cu}$, and $\mathrm{W}$ and disks of quartz and various kinds of porcelain. Strong $\mathrm{Si}$ II-IV and $\mathrm{O}$ II-VI lines are produced by means of a quartz disk with appropriate metal electrodes.

The same arrangement was used by Toresson [37, 38], who produced Si III and Si iv by means of a quartz disk between silicon electrodes. Since silicon is a poor conductor at ordinary temperatures, the spark frequency is kept high enough to reach a temperature where the material becomes conducting. Boron also is a very poor conductor at temperatures below some hundreds of degrees centigrade. In order to produce the B III spectrum, Gunnvald [39] uses conducting electrodes prepared in the following way. A mixture of boron and aluminum powder is pressed in a mold and heated to a temperature above the melting point of aluminum. In combination with a quartz disk of Bockasten's design, these electrodes produced new B II and B III lines. The excitation of B III requires a high condenser voltage and a small inductance and this results in diffuse lines.

The sliding spark arrangement used by Sugar [40] to excite spectra of ionized rare earths, works at much lower voltage. The spark chamber contains helium at a pressure of 30 torr, and disks of the metal to be investigated serve as electrodes separated about $3 \mathrm{~mm}$ by a lavite spacer. The spark gap is in series with a $10 \mu \mathrm{F}$ capacitor, a damping resistor, a rotating spark gap, and an inductor. With a $1000 \mathrm{~V}$ charging voltage and a $500 \mu \mathrm{H}$ inductance a fully developed $\operatorname{Pr}$ III spectrum was obtained. With no inductor, the same charging voltage, and a suitable damping resistance, the Pr IV spectrum was enhanced. Critica! damping was found to suppress Pr II better than an oscillatory discharge.

In order to separate Ce II from Ce III, Sugar [41] has used larger inductances - about $1000 \mu \mathrm{H}$ - and lower voltages (down to $300 \mathrm{~V}$ ), thus producing the Ce II spectrum and suppressing Ce III. The arrangements allow a very good separation of ionization stages.
Low-inductance sliding spark sources have been designed recently for the excitation of higher ionization stages [42], Bogen and Conrads [43] (containing further references) having obtained strong $\mathrm{C} \mathrm{v}$ and $\mathrm{C}$ vi spectra. In these experiments the inductance is in the 5 to $20 \mathrm{nH}$ range.

The use of the sliding spark is not limited to solid materials. It is also possible to excite gases by letting them in through a bore in one of the electrodes, in accordance with the arrangement used for vacuum sparks by Phillips and Parker [44]. This has been done by Toresson [45] and by Bockasten, Hallin, and Hughes [46]. The former has obtained spectra of $\mathrm{S}_{\mathrm{I}-\mathrm{S}}$ VI and $\mathrm{O}_{\mathrm{I}}-\mathrm{O}$ VI, and the most easily excited $K r$ viII lines in the vacuum ultraviolet; the latter authors report the observation of a strong Ne iv and a weak Ne v spectrum.

\section{Comparison Between High-Frequency Excitation and Excitation by Sliding Sparks}

The high-frequency method can be used for the excitation of gases as well as of vapors from liquids and solids provided that the vapor pressure is sufficiently high at the working temperature of the discharge tube. The sliding spark method was originally developed for the excitation of solid materials but was later found to be suitable for gases and vapors as well. As regards high-frequency excitation, we will confine ourselves to the electrodeless discharge. High-frequency pulses from existing tube oscillators can produce the fourth spectra of argon, krypton, and xenon. Since the analysis is not finished it is not possible at the moment to tell to what completeness these spectra are developed. Boyce reports still higher ionization stages obtained by spark-generated oscillations, namely $\mathrm{Ar} \mathrm{v}$; as already pointed out, the observed $\mathrm{Ar} \mathrm{v}$ lines are transitions to the ground term from the lowest excited states and so are those most easily excited. The excitation by means of sliding sparks gives still higher ionization stages - the eighth spectrum is reported in one case.

As regards line quality, a just comparison between the electrodeless high-frequency and the sliding spark methods would require the observation of the same lines by means of both methods and, preferably, with the same kind of high-dispersion spectrographs. In the absence of such careful observations, the comparison must be approximate. Experience from the investigation of ionized inert gases shows that high frequency pulses give very good lines; even the $n f-n g$ lines are sharp. In that respect the highfrequency excitation is believed to be superior to the sliding spark, which produces Stark-effect shifts of sensitive levels. It should be easier to avoid impurity lines at high-frequency excitation. Although the separation of lines belonging to different ionization stages can be done in both cases, the highfrequency excitation method is probably superior.

In fact, the two methods complement each other. Roughly speaking, the excitation by means of high-frequency pulses is recommended for gaseous 
elements or compounds and vapors up to about the third ionization stage. The sliding spark should be used for the excitation of solid elements and, also, for gases in order to reach still higher ionization stages.

The present review was originally prepared for internal use at the National Bureau of Standards. It is published in the hope that it may have a somewhat wider interest in spite of its limited scope.

\section{References}

[1] de Bruin, T. L., Zeit. Phys. 46, 856 (1928).

[2] de Bruin, T. L., Zeeman Verhandlingen p. 413 (1935).

[3] Humphreys, C. J., Phys. Rev. 47, 712 (1935).

[4] Paul, F. W., and Polster, H. D., Phys. Rev. 59, 424 (1941).

[5] de Bruin, T. L., Physica 3, 809 (1936).

[6] Rao, A. B., and Krishnamurty, S. G., Proc. Phys. Soc. 51, $772(1939)$.

[7] Humphreys, C. J., unpublished (1953).

[8] Thomson, J. J., Phil. Mag. 32, 321 (1891).

[9] Bloch, L., and Bloch, E., J. Phys. Radium 4, 333 (1923).

[10] Bloch, L., Bloch, E., and Déjardin, G., Ann. Phys. (Paris) 1-2, 461 (1924).

[11] Boyce, J. C., Phys. Rev. 46, 378 (1934).

[12] Boyce, J. C., Phys. Rev. 48, 718 (1935).

[13] Boyce, J. C., Phys. Rev. 48, 396 (1935).

[14] Boyce, J. C., Phys. Rev. 49, 730 (1936); also unpublished (1936).

[15] Eriksson, K. B., Ark. Fys. 13, 303 (1958).

[16] Bockasten, K., Hallin, R., Johansson, K. B., and Tsui, P., Physics Letters 8, 181 (1964).

[17] Esclangon, F., Ann. Phys. (Paris) 1, 267 (1934).

[18] Gatterer, A., and Frodl, V., Ricerche Spettroscopiche 1, 201 (1946).

[19] Goudet, G., Herreng, P., and Nief, G., Compt. Rend. 214, 62 (1942).
[20] Roig, J., and Buret, A., Compt. Rend., 230, 1394 (1950).

[21] Minnhagen, L., and Stigmark, L., Ark. Fys. 8, 471 (1954).

[22] Minnhagen, L., and Stigmark, L., Ark. Fys. 13, 27 (1957).

[23] Minnhagen, L., Stigmark, L., and Petersson, B., Ark. Fys. 16, 541 (1960).

[24] Stigmark, L., to be published.

[25] Kaufman, V., and Andrew, K. L., J. Opt. Soc. Am. 52, $1223(1962)$.

[26] Minnhagen, L., J. Opt. Soc. Am. 54, 320 (1964).

[27] Meggers, W. F., and Westfall, F. O., J. Res. NBS 44, 447 (1950).

[28] Jacobsen, E., and Harrison, G. R., J. Opt. Soc. Am. 39, 1054 (1949).

[29] Zelikoff et al., J. Opt. Soc. Am. 42, 818 (1952).

[30] Corliss, C. H., Bozman, W. R., and Westfall, F. O., J. Opt. Soc. Am. 43, 398 (1953).

[31] Tomkins, F. S., and Fred, M., J. Opt. Soc. Am. 47, 1087 (1957).

[32] Worden, E. F., Gutmacher, R. G., and Conway, J. G., Appl. Optics 2, 707 (1963).

[33] Vodar, B., and Astoin, N., Nature 166, 1029 (1950).

[34] Roman, J., and Vodar, V., Spectrochim. Acta 8, 229 (1956).

[35] Bockasten, K., Ark. Fys. 9, 457 (1955).

[36] Bockasten, K., Ark. Fys. 10, 567 (1956).

[37] Toresson, Y., Ark. Fys. 18, 389 (1961).

[38] Toresson, Y., Ark. Fys. 17, 179 (1960).

[39] Gunnvald, P., unpublished.

[40] Sugar, J., J. Opt. Soc. Am. 53, 831 (1963).

[41] Sugar, J., J. Opt. Soc. Am. (in press) (1964).

[42] Conrads, H., Diplomarbeit (Aachen 1961).

[43] Bogen P., and Conrads, H., Compt. Rend. de la VIe Conférence Internationale sur les Phenomènes d'Ionisation dans les Gaz, III, 337 (Paris 1963).

[44] Phillips L. W., and Parker, W. L., Phys. Rev. 60, 301 (1941).

[45] Toresson, Y., Thesis, Lund (1960).

[46] Bockasten, K., Hallin, R., and Hughes, T. P., Proc. Phys. Soc. 81, 522 (1963).

(Paper 68C4-169) 